\title{
Analysis on Passenger Distribution Based on Precise Comprehensive Traffic System
}

\author{
Yang jie ${ }^{1}$, Wang jie ${ }^{2,3}$ \\ ${ }^{1}$ Zhejiang Institute of Communications, Hangzhou, China \\ ${ }^{2}$ Zhejiang Scientific Research Institute of Transport, Hangzhou, China \\ ${ }^{3}$ National Public Information Platform for Transportation and Logistics, Hangzhou, China \\ Email address: \\ yangjie@zjvtit.edu.cn (Yang jie)

\section{To cite this article:} \\ Yang jie, Wang jie. Analysis on Passenger Distribution Based on Precise Comprehensive Traffic System. International Journal of Science, \\ Technology and Society. Vol. 4, No. 5, 2016, pp. 74-79. doi: 10.11648/j.ijsts.20160405.12
}

Received: September 26, 2016; Accepted: October 13, 2016; Published: October 17, 2016

\begin{abstract}
Building a comprehensive traffic system is a new pattern and also a necessary choice of China's traffic development. Based on the background of urban mass construction of rail transit, this paper takes the hidden danger of traffic system as a breakthrough point, focusing on secure guarantee measures, and come up with an idea that we should take the coordination and cooperation between road transit and rail transit as well as internal system of rail transit in the public traffic system as a breakthrough, and build a precise urban comprehensive traffic system. The author has also done a quantitative research on scale of passenger flow which is influenced by operation failures in rail transit network, which is regarded as a key problem of precise comprehensive traffic system.
\end{abstract}

Keywords: Traffic Engineering, Precise, Comprehensive Traffic, Collaborative Roads and Rails

\section{Introduction}

Transportation is the foundation of modern economic society's development, and whether it's well developed has a direct effect on the support of economic development, people's quality of life and so on. Up to now, transportation in China hasn't made great progress. There is still a certain gap between the total scale and quality of transportation and the demand of China's economic and geographic development, which results in failing to meet the demand of industrialization and urbanization in China and also failing to provide powerful support for the development of economic society. Meanwhile, transportation is an industry that takes more resources and energy. While it brings humans benefits and help them to remove the obstacles of space, it also brings some negative influences such as occupying lots of land resources, having a side effect on environment etc. Especially in big cities and megacities, per capita resource capacity and environmental capacity are far lower than the standard of developed countries, even lower than the average level of world and the development of transportation is strongly constrained by resources. Therefore, precise development of comprehensive traffic system is to develop more quickly and use the limited resources more effectively, which means that under the circumstances of affordable resource consumption and costs, it can establish an effective comprehensive traffic system to better meet the demand of people's travelling and create more comfortable living and working environment. Coexistence and tight cooperation of all kinds of transportation means in comprehensive traffic system will bring a more advanced traffic system and build a more solid foundation to improve the travel efficiency. Accordingly, comprehensive traffic system is not only compliant with the development of economic society, but also a necessary outcome of the transportation development.

\section{Analysis of Collaborative Tracks' Role in Comprehensive Traffic System}

\subsection{Analysis of Development Trend of Comprehensive Traffic System}

Comprehensive traffic system is a transportation synpaper in which within the socialized scope and unified process of transportation, various means of transportation are in division 
and cooperation, organic combination, continuity and reasonable layout based on their respective technical and economic features. It consists of railways, highways, waterways, pipelines, airlines and all kinds of means of transportation as well as their lines and stations. Comprehensive traffic system is generally made up of three systems: the first one is the integrated transport network with certain technical equipment and its junction system; the second is the transport system combined by all the means of transportation; the last one is comprehensive traffic system of management, organization and coordination.

Building a comprehensive traffic system is the new trend and direction in contemporary traffic development. There are two major trends in contemporary traffic development: one is that with the development of world's new technology revolution, transportation is widely use the new technology to realize the modernization of means and equipment of transport; the other is that with diversification of transport means and the unification of transport process, all kinds of transport means develop in the direction of division, cooperation and coordination. Within the scope of world, these two trends form a new direction of development of contemporary transportation.

Building a comprehensive traffic system is a new pattern and also a necessary choice of China's traffic development. Building a comprehensive traffic system can enhance the effective transport productivity, and relieve the traffic pressure. Transportation is such a big system that every transport mean, line and link need to be coordinated, otherwise it can't give full play to effective transport productivity. For years, transportation in China is under imbalanced development. For example, some lines bear huge transport pressure, but others can't be fully used; some transport means are heavily overloaded, while others can't play their full role. Adopting the comprehensive traffic system will effectively solve the current problem of disharmony and imbalance. Building a comprehensive traffic system is a significant way to improve the economic efficiency of transport. According to the technical features of each transport mean, it sets up the reasonable transport structure, so that all kinds of transport means can make best use of their advantages and bypass the disadvantages and then it can enlarge transport capacity as well as improve economic performance.

\subsection{Importance of Rail Transit System in Urban Comprehensive Traffic System}

Railway system and routine bus system are the main modes in urban public traffic system especially in big cities. From the points of urban traffic demand and economic development, mass construction of subway is optimal for the government to solve public traffic problems. To accelerate the construction of public traffic as the main target, and the urban motorized travel system formed by rail transit network, public bus and tramcar etc. has been a main idea for big cities to deal with the traffic jam. Among the major big cities in the world, rail transit capacity accounts for more than $50 \%$ of public traffic capacity, some even more than $70 \%$. For example, Paris, a city with the population of 10 million, annual passenger traffic capacity is 1.2 billion, among which rail transit bears $70 \%$ of public traffic capacity; and this percentage in Moscow is 55\%. In London, there are 9 metro lines, whose total length is $500 \mathrm{~km}$ and the daily traffic capacity is 3 million, which can meet the travel demand of $40 \%$ of the people. In metropolitan area of Tokyo, Japan, metro lines cover more than $280 \mathrm{~km}$, and the daily traffic capacity of rail transit is more than 30 million, accounting for $86 \%$ of total passenger traffic capacity.

In urban comprehensive traffic system, rail transit, as the main framework, plays a key role in urban normal operation. To build a comprehensive traffic system with precise development of collaborative tracks and to accelerate the construction of public traffic as the main target, and the urban motorized travel system formed by rail transit network, public bus and taxi etc. have been a main idea for big cities in China to deal with the traffic jam. Facing the actual needs, urban comprehensive traffic system should not only attach importance to discussion of theory and idea, but should also focus on the research of enforceable methods and strategies.

In the megacities and big cities of China, as the expansion of rail network's scale and complexity of its pattern, the influence that rail transit and surface traffic as well as all lines of rail transit network bring out shows the trend of diversity, intension and enlargement. Take Hangzhou as an example. In the past two years, bad weather especially the heavy precipitation has led to several emergencies which have huge impacts on urban traffic. When handling these emergencies, it's obvious to find out that there are many defects of coordination among traffic means in urban comprehensive traffic system. A more important reason is the lack of concrete plans and technology of comprehensive traffic, which makes it difficult to implement the policies. In general, comprehensive traffic system is still in a stage of a concept, and the applied methods haven't been further studied. In a word, there is a long distance that building a precise comprehensive traffic system needs to cover.

\subsection{Analysis of Present Status of Rail Transit in CHINA}

As what has stated above, rail transit plays a major role in urban comprehensive traffic system and has a tremendous influence on urban normal operation. The construction of urban rail transit is crucial to ease traffic pressure brought by urbanization, which is exactly the reason why rail transit develops at an amazing speed in all big cities of China. From 1995 to 2008, in these 12 years, the cities in China that built rail transit had increased from 2 to 10 and the investment had been put in as the speed of more than RMB 10billion annually; At the end of 2009, the State Council approved the subway construction planning of 22 cities; In 2012, after China carried out policy of "steady growth", 14 cities including Changzhou, Xiamen, Shenyang and so on were approved for their subway plans in a short term or adjustments of plans, totally covering a distance of $993 \mathrm{~km}$. By the end of December, 2015, there were 110 lines of rail transit in 25 cities of China that had been completed and put into operation, including 2257 stations, the total operating mileage reaching $3286.51 \mathrm{~km}$. Statistics of total 
mileage and stations of all rail transit operating lines in cities of China are shown in table 1.

Table 1. Statistics of total mileage and stations of all rail transit operating lines in cities of China.

\begin{tabular}{|c|c|c|c|c|c|c|c|c|}
\hline \multirow{2}{*}{ No. } & \multirow{2}{*}{ City } & \multirow{2}{*}{$\begin{array}{l}\text { Total Operating } \\
\text { Lines }\end{array}$} & \multicolumn{4}{|c|}{ Type of Lines } & \multirow{2}{*}{ Stations } & \multirow{2}{*}{$\begin{array}{l}\text { Total mileage } \\
(\mathbf{k m})\end{array}$} \\
\hline & & & Subway & Light rail & Tramcars & magnetic suspension & & \\
\hline 1 & Shanghai & 16 & 588.64 & & 10.00 & 30 & 369 & 628.64 \\
\hline 2 & Beijing & 18 & 553.39 & & & & 336 & 553.39 \\
\hline 3 & Guangzhou & 10 & 247.02 & 3.94 & 7.70 & & 170 & 258.66 \\
\hline 4 & Nanjing & 7 & 232.25 & & 7.76 & & 137 & 240.01 \\
\hline 5 & Chongqing & 4 & 110.32 & 86.97 & & & 121 & 197.29 \\
\hline 6 & Shenzhen & 5 & 177.58 & & & & 131 & 17587. \\
\hline 7 & Dalian & 7 & 42.20 & 88.45 & 40.38 & & 100 & 171.03 \\
\hline 8 & Tianjin & 6 & 94.16 & 45.41 & 7.86 & & 100 & 147.43 \\
\hline 9 & Wuhan & 4 & 126.03 & & & & 102 & 126.03 \\
\hline 10 & Shenyang & 6 & 60.26 & & 56.00 & & 134 & 116.26 \\
\hline 11 & Chengdu & 3 & 88.44 & & & & 70 & 88.44 \\
\hline 12 & Hangzhou & 3 & 81.56 & & & & 56 & 81.56 \\
\hline 13 & Suzhou & 3 & 52.34 & & 18.19 & & 57 & 70.53 \\
\hline 14 & Changchun & 4 & 16.33 & 31.99 & 17.22 & & 82 & 65.54 \\
\hline 15 & Kunming & 3 & 60.99 & & & & 35 & 60.99 \\
\hline 16 & Wuxi & 2 & 56.16 & & & & 46 & 56.16 \\
\hline 17 & Xi'an & 2 & 52.00 & & & & 40 & 52.00 \\
\hline 18 & Ningbo & 2 & 49.23 & & & & 42 & 49.23 \\
\hline 19 & Nanchang & 1 & 28.70 & & & & 24 & 28.70 \\
\hline 20 & Changsha & 1 & 27.05 & & & & 23 & 27.05 \\
\hline 21 & Zhengzhou & 1 & 25.41 & & & & 20 & 25.41 \\
\hline 22 & Huai'an & 1 & & & 20.07 & & 23 & 20.07 \\
\hline 23 & Harbin & 1 & 17.73 & & & & 18 & 17.73 \\
\hline 24 & Foshan & 1 & 14.78 & & & & 11 & 14.78 \\
\hline 25 & Qingdao & 1 & 12.00 & & & & 10 & 12.00 \\
\hline
\end{tabular}

\section{Analysis of Key Problem of Collaborative Tracks}

The construction of rail transit is in full swing and through this phenomenon, we must figure out that operation of rail transit has its own special security risk and huge impacts. Considering security and reliable operation of future urban traffic, we must make a careful and thorough plan for the operation and management of rail transit, such a special transportation means.

From the view of security of future urban traffic, urban public traffic security is the magnitude guarantee to ensure urban normal operation. In the Outline of the National Program for Long-and Medium-Term Scientific and Technological Development (2006-2020), "public security" is one of 11 key areas and "early warning and rescue of major production accidents" is one of 68 optimal subjects. Rail transit is a typical mode constrained by space, but rail transit integrated hub is the most important operating carrier and basis of urban rail transit. Meanwhile, it's an area where urban public traffic is the most active and security flaws and safety production conflicts are the most concentrated. Safety production has the characteristics of difficult technology and extensive damage, so it's a great responsibility to ensure the safety of subway operation.

As for the rail transit network, with the rapid expansion of the scale of the urban rail network, accessibility and the travel stability of rail transit system are continuously improving, at the same time, all uncertain factors that influence normal operation of rail network system are increasing. Emergencies result in an abnormal decline of transport capacity. Especially when operation is interrupted, there will be a greater impact on urban traffic system.

From each node of rail transit network, stations of modern urban rail transit generally have the characteristics of large volume, complex structure, large passenger flow and high activity intensity. Operating pressure brought about by normal large passenger flow as well as the destruction of all kinds of emergencies has greater influence.

When combining road transit with rail transit, it's necessary to ensure all factors such as scale, degree and distribution which are all influenced by network, so that we can come up with comprehensive strategies and methods to deal with emergencies. Owing to the differences of space shape, structure and environment, from all aspects of nature, form and degree, different types of emergencies in different areas have different impact models, evolution features and scale distributions. What's more, forms of changing and combination are various, thus, it's more difficult to judge the level of emergency. In practice, "large scale, wide range" is always taken to describe the emergency, but such a description can't make it more clear and accurate, which is not good to handle the emergency.

In the megacities of China, as scale of rail network expands and pattern of rail network becomes increasingly complex, forms of influence among lines become more diverse, and its scale also expands. Moreover, parts of built lines have already been in a stage of aging equipment and frequent failures, and new lines will have the same problems several years later. Because of the special characteristics of 
rail transit such as operation constrained by space, highly centralized control and large passenger flow, there is a pressing need to pay more attention to secure operation of subway. Accordingly, it's necessary to do the research on characteristics and mechanism of every emergency of urban rail transit hub. Also, it's important to conduct a survey about such a significant practical problem, establishing safety emergency management of urban rail station.

When sector operation of rail transit is interrupted by all types of risks, a large number of passengers are detained outside the station. And things often get worse if there are other unfavorable factors such as bad weather. To deal with such a typical emergency of passengers' detention caused by sector operation interruption, we need the coordination and cooperation of all units of both surface road transit and rail transit in order to quickly take practical and feasible measures. In special, it's key to keep the link of rail transit and road transit, and ensure that bus can connect well and safe and timely evacuation of passenger flow, try to maintain order of network operation, so that negative impact on the public caused by improper measures and inaccurate information can be avoided. For example, when rail transit interrupts, we can arrange some short routings and make these short routings to shorten the range of interruption. In this premise, bus shuttle can be a necessary supplement. Such a series of solutions are all the important contents of coordination and cooperation between road transit and rail transit.

Then, in real life, in recent years, in several big cities of China, many emergencies resulted from bad weather especially heavy precipitation has a great impact on urban traffic. Emergency response to these emergencies reveals many defects of coordination and cooperation among all means in urban comprehensive traffic system. One of more significant reasons is the lack of concrete plans and technology of comprehensive traffic, which makes it difficult to implement the policies. In general, comprehensive traffic system is still in a stage of a concept, and the applied methods haven't been further studied. In a word, there is a long distance that building a precise comprehensive traffic system needs to cover.

Facing the actual needs, urban comprehensive traffic system should not only attach importance to discussion of theory and idea, but should also focus on the research of enforceable methods and strategies. Based on the background of urban mass construction of rail transit, this paper takes the hidden danger of traffic system as a breakthrough point, focusing on secure guarantee measures, and come up with an idea that we should take the coordination and cooperation between road transit and rail transit as well as internal system of rail transit in the public traffic system as a breakthrough, and build a precise urban comprehensive traffic system. The author has also done a quantitative research on scale of passenger flow which is influenced by operation failures in rail transit network, which is regarded as a key problem of precise comprehensive traffic system.

\section{Analysis of Passenger Flow Aggregation Based on Precise Comprehensive Traffic System}

\subsection{Systematic Analysis and Parameter Design}

When traffic operation failures result in decline of effective transport capacity, the most intuitive phenomenon is the increasing number of delayed passengers in station. In order to show the changes of the delayed passengers, we need to figure out the changing relationship among passenger flow, transport capacity and delayed passenger. First, set definitions on a group of parameters, and suppose that there is a station " $n$ " within " $\mathrm{t}$ " hours.

$P I_{n}^{t}$ : Total volume of inbound passengers ( $P I U$ and PID respectively represent inbound passenger volume from and to the terminal)

$P O_{n}^{t}$ : Total volume of outbound passengers ( $P O U$ and $P O D$ respectively represent outbound passenger volume from and to the terminal)

$Q U_{n}^{t}$ : Total volume of passenger that could be carried in theory from the terminal

$Q D_{n}^{t}$ : Total volume of passenger that could be carried in theory to the terminal

$Q U A_{n}^{t}$ : Total volume of passenger that could be in fact accepted from the terminal (Saturation function)

$Q D A_{n}^{t}$ : Total volume of passenger that could be in fact accepted to the terminal (Saturation function)

$R Q_{n}^{t}$ : Total volume of delayed passenger

$R I_{n}^{t}$ : Growth of delayed passenger (Condition function)

Thereinto, $Q U A_{n}^{t}$ and $Q D A_{n}^{t}$ are determined according to service level and set full-load ratio. $R I_{n}^{t}$ indicates the effective transport capacity and change rate of passenger flow.

In addition, give definitions to $\mathrm{TC}$ and $\mathrm{TS}$ which are respectively regarded as auxiliary parameters of interval of the train and time of stopping.

Influence on passenger should include scale and degree these two parts. Moreover, scale indicates the volume of influenced passengers, and degree indicates the duration. Measure for the scale can be the numbers, and for the degree can be the waiting time. However, when the operation is poor, the individual waiting time of passengers will be different and $Q U A_{n}^{t}$ in every interval of operation is also different, so waiting time at any time should be the cumulative sum of waiting time of all the passengers who were then in the station. This is a problem that haven't been taken into consideration before. According to general queuing theory model, we can suppose that every inbound passenger at different times all obeys the queuing service rule and scale of the delayed is function of arrival rate of passenger $P I U_{n}^{t}$ and service rate of train $Q U A_{n}^{t}$, that is, $R Q_{n}^{t}=f\left(P I U_{n}^{t}\right.$, 
$\left.Q U A_{n}^{t}\right)$. As shown in formula (3.1)

$$
R Q_{n}^{t}=\left(P I U_{n}^{t}-Q U A_{n}^{t}\right)+R Q_{n}^{t-1}=(T C+T S) \cdot \sum_{t=0}^{k}\left[P I U_{n}^{t}-Q U A_{n}^{t}\right]
$$

$P W T$ indicates waiting time of all delayed passengers in specific interval, and its value is only related to scale of the delayed and operation interval of train, that is, $P W T$ $=f\left(R Q_{n}^{t}, T C, T S\right)$. Waiting time of passenger can be normally shown in formulas (2) and (3), which respectively represent specific stations and waiting time of the delayed of related stations, these two forms.

$$
\begin{gathered}
P W T_{t}=R Q_{n}^{t} \cdot(T C+T S) \\
P W T_{t}=\sum_{i=1}^{\mathrm{n}} R Q_{n}^{t} \cdot(T C+T S)
\end{gathered}
$$

\subsection{Modeling on Degree of Influence on Passengers}

Serving the time of entering stop as a division, the waiting time of each group consisting of the same individuals is only in connection with the delayed passenger volume $R Q_{n}^{t}$ when $t=k, Q U A_{n}^{t}$ when $t>k$ and $T C, T S$, but it isn't related to the $P I U_{n}^{t}$ when $t>k$. Supposing that the meaning of $P I U_{n}^{t}$ is invariant and $Q U A_{n}^{t}$ indicates the service rate of train, then give a definition to a cumulative time index $Q T W$ in this paper to represent such degree of influence of delayed passengers and still take train from the terminal as an example, we can start modeling and the process is shown as Step1- Step4:

Step1 $P I U_{n}^{t}$ and $Q U A_{n}^{t}$ respectively refer to in operation interval when $t=k$ the arrival rate of passenger and effective transport capacity which indicates the acceptable passenger volume of the train relying on transport capacity, specific full-load rate and actual passenger volume. When $t=k$, the delayed passenger volume is $R Q_{n}^{t}$, as shown in formula (4)

$$
R Q_{n}^{k}=\sum_{t=1}^{n}\left(P I U_{n}^{t}-Q U A_{n}^{t}\right)
$$

Step2 According to the queuing rule, duration from when passenger enters into station to when passenger travels away only depends on the delayed passenger volume $R Q_{n}^{k}$, and the $Q U A_{n}^{t}$ when $t>k$ as well as $T C$ and $T S$, but it has nothing to do with $P I U_{n}^{t}$.

$$
\begin{gathered}
\sum_{t=k+1}^{p} Q U A_{n}^{t} \cdot(T C+T S)=\sum_{t=0}^{k}\left[P I U_{n}^{t}-Q U A_{n}^{t}\right] \cdot(T C+T S)+1 \\
Q T W=(p-k-1) \cdot(T C+T S)
\end{gathered}
$$

Step3 During the interval time $t$ of operation of any trains, the sum for total number $R Q_{n}^{k}$ of waiting time of all passengers can be represented by $Q T W T$.

$$
Q T W T=\sum_{i=1}^{R Q_{n}^{k}} Q T W_{i}
$$

Step 4 The calculation of $Q T W T-P W T$ can get a new index which is represented by $Q T W_{R}$ and whose definition is cumulative time of delayed passengers. This index is used to describe the degree of influence that declined effective transport capacity has on the passengers. The concrete implications are as follows.

(a) when $Q T W_{R}=0$, it refers that an interval of operation equals to the waiting time from when passenger enters into station to when passenger travels away;

(b) when $Q T W_{R}>0$, the waiting time of some passengers is more than an interval of operation, so there is a delayed phenomenon. Just as shown in formula (8)

$$
Q T W_{R}=Q T W T_{t}-P W T_{t}=\sum_{i=1}^{R Q_{n}^{k}} Q T W_{i}-\sum_{i=1}^{t} R Q_{n}^{t} \cdot(T C+T S)
$$

In the formula (8), $Q T W T$ indicates the sum of waiting time of all passengers, and the formula $Q T W_{R}=Q T W T-$ $P W T$ is used to calculate whether waiting passengers would wait for several intervals. If the value of $Q T W_{R}$ is small, it indicates that $Q U A_{n}^{t}$ evacuates passengers effectively in prevention of delaying, and the waiting interval is small caused by influenced passengers; while if it's large, it indicates that there are passengers who need to wait for other trains. Meanwhile, because the calculation of $Q T W_{R}$ is based on the cumulative value, although there are a few passengers waiting, the $Q T W_{R}$ can also clearly reflect the sum of actual waiting time of every passenger. However, the calculation of QTWT can only show the scale of the delayed indirectly, and it can neither reflect the actual waiting time of passengers nor measure the degree of influence on passengers when the operation is poor.

What has to explain is that the calculation for the total waiting time of passengers is just to measure the degree of influence and when set up the model, the assumption that all passengers obey the queuing service rule at any time is not a truth in a reality, but the method and thinking of the calculation can still be applied. When evaluating on virtual condition, even though there may be some passengers who don't obey the queuing service rule, the total waiting time is still invariant. What just changes are that the cost of this waiting time is undertaken by passengers who obey the queuing service rule.

\subsection{Parameter Relations of Passengers' Influence}

Transport capacity here refers to the acceptable volume of passengers when the train pulls in, which depends on the maximum number of passengers of the train and the level of service. Taking the train from the terminal as an example, the 
major parameter relations are as follows:

$$
\begin{gathered}
R Q_{n}^{t}=\left(P I U_{n}^{t}-Q U A_{n}^{t}\right)+R Q_{n}^{t-1} \\
R I_{n}^{t}= \begin{cases}P I U_{n}^{t}-Q U A_{n}^{t} & \text { if } P I U_{n}^{t}>Q U A_{n}^{t} \\
0 & \text { if } P I U_{n}^{t} \leq Q U A_{n}^{t}\end{cases}
\end{gathered}
$$

$Q U A_{n}^{t}$ meets the following constraints, and it's in fact a saturation function.

$$
\begin{gathered}
\min \left\{P I U_{n}^{t}+\sum_{t=0}^{k-1}\left[P I U_{n}^{t}-Q U A_{n}^{t}\right], Q U A_{n}^{t}\right\} \geq Q U A_{n}^{t} \\
\geq \max \left\{P I U_{n}^{t}+\sum_{t=0}^{k-1}\left[P I U_{n}^{t}-Q U A_{n}^{t}\right], S Q S\right\} \geq 0 \\
\text { sat. } Q U A_{n}^{t}= \begin{cases}\min Q U A_{n}^{t} & \text { if } Q U A_{n}^{t}<\min Q U A_{n}^{t} \\
\max Q U A_{n}^{t} & \text { if } Q U A_{n}^{t}>\max Q U A_{n}^{t} \\
Q U A_{n}^{t} & \end{cases}
\end{gathered}
$$

\section{Conclusion}

Each traffic poor operation differs in time, place, propagation characteristics and degree of influence, and they are also influenced by different replacements and travel means, so that the changing process of delayed passengers will be diversified. On this basis, we need to overall think and discuss the evolution model of spreading on a level of rail network and the related quantitative analysis methods.

The construction of precise urban comprehensive traffic system is still a relatively new area of research, which involves many details that need to deal with in the coordination of multisystem. The future research should pay attention to the technology, management and other weaknesses. Conduct a research and take related actions, and finally develop a systematic, complete and operable technical system.

\section{Foundation}

In-aid Program of Education Department of Zhejiang Province (Y201329709)

\section{References}

[1] Jin Jia. Analysis on Concept of Comprehensive Transportation [J]. Comprehensive Transportation, 2011, 7:4-7.

[2] Ping Guo. Characteristics and Prediction for Related Problems of Passenger Flow in Urban Rail Transit [J]. Research on Urban Rail Transit, 2010, 13 (1):58-62.

[3] Yi Wang, Ruihua Xue. Forcast of Short-term Metro Passenger Flow Based on the Periodically Varying Characteristics [J]. Research on Urban Rail Transit, 2010, 13(1):46- 49.

[4] Jian Li. Passenger Distribution Modeling and Security Alarms Research under Continuous Delayed Capacity Declined Station for Subway Station [C]. The key technology of city rail transit forum Chinese in 2013, 2013.09

[5] E. Wendler. The scheduled waiting time on railway lines [J]. Transportation Research Part B, 2007, 41: 148-158.

[6] M. Pavankumar, M. M. Dessouky and O. Fernando, et al. A delay estimation technique for single and double-track railroads [J]. Transportation Research Part E, 2009, 4: 1-13.

[7] T. Huisman, R. J. Boucherie. Running times on railway sections with heterogeneous train traffic [J]. Transportation Research Part B, 2001, 35:271-292.

[8] Q. Lu, M. M Dessouky, R. C. Leachman. Modeling of train move-ments through complex networks [J]. ACM Transactions on Model-ing and Computer Simulation, 2004, 14:48-75.

[9] M. Pavankumar, M. M. Dessouky and O. Fernando, et al. A delay estimation technique for single and double-track railroads [J]. Transportation Research Part E, 2009, 4:1-13.

[10] A. Higgins, E.Kozan. Modeling train delays in urban networks [J]. Transportation Science, 1998, 32 (4):346-357. 